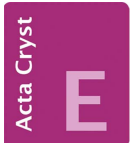

CRYSTALLOGRAPHIC COMMUNICATIONS

ISSN 2056-9890

Received 5 July 2018

Accepted 24 July 2018

Edited by M. Weil, Vienna University of

Technology, Austria

Keywords: crystal structure; transition metal vanadate; solid-state reaction; alluaudite structure type.; crystal structure.

CCDC reference: 1857879

Supporting information: this article has supporting information at journals.iucr.org/e

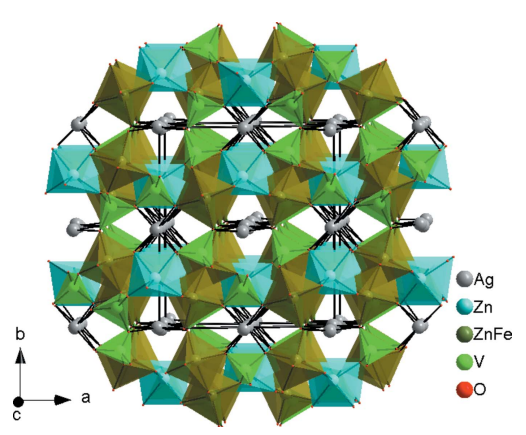

OPEN $\odot$ ACCESS

\section{Crystal structure of disilver(I) dizinc(II) iron(III) tris(orthovanadate) with an alluaudite-type structure}

\author{
Nour El Houda Lamsakhar,* Mohammed Zriouil, Abderrazzak Assani, Mohamed \\ Saadi and Lahcen El Ammari
}

Laboratoire de Chimie Appliquée des Matériaux, Centre des Sciences des Matériaux, Faculty of Sciences, Mohammed V University in Rabat, Avenue Ibn Batouta, BP 1014, Rabat, Morocco. *Correspondence e-mail: nlamsakhar@gmail.com

The title compound, $\mathrm{Ag}_{2} \mathrm{Zn}_{2} \mathrm{Fe}\left(\mathrm{VO}_{4}\right)_{3}$, has been synthesized by solid-state reactions and belongs to the alluaudite structure family. In the crystal structure, four sites are positioned at special positions. One silver site is located on an inversion centre (Wyckoff position $4 b$ ), and an additional silver site, as well as one zinc and one vanadium site, on twofold rotation axes $(4 e)$. One site on a general position is statistically occupied by $\mathrm{Fe}^{\mathrm{III}}$ and $\mathrm{Zn}^{\mathrm{II}}$ cations that are octahedrally surrounded by $\mathrm{O}$ atoms. The three-dimensional framework structure of the title vanadate results from $\left[(\mathrm{Zn}, \mathrm{Fe})_{2} \mathrm{O}_{10}\right]$ units of edge-sharing $\left[(\mathrm{Zn}, \mathrm{Fe}) \mathrm{O}_{6}\right]$ octahedra that alternate with $\left[\mathrm{ZnO}_{6}\right]$ octahedra so as to form

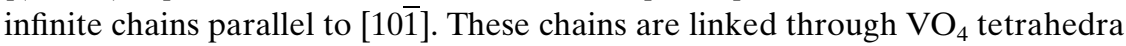
by sharing vertices, giving rise to layers extending parallel to (010). Such layers are shared by common vanadate tetrahedra. The resulting three-dimensional framework delimits two types of channels parallel to [001] in which the silver sites are located with four- and sixfold coordination by oxygen.

\section{Chemical context}

The crystal structure of the mineral alluaudite with general formula $A(1) A(2) M(1) M(2)_{2}\left(X \mathrm{O}_{4}\right)_{3}$ was determined nearly fifty years ago by Moore (1971). In the structure, the two $A$ sites can be occupied by mono- or divalent cations of medium size, and the $M(1)$ and $M(2)$ sites can accommodate di- or trivalent cations, which are generally transition metals and are octahedrally surrounded. The specific feature of the alluaudite structure is the existence of two channels parallel to [001] in which the $A$-site cations are located. As a result, alluauditetype compounds can exhibit electronic and/or ionic conductivity (Hatert, 2008). In addition, alluaudite-type compounds have been reported as materials for fossil energy conversion, as sensor materials and storage energy materials (Korzenski et al., 1998), and as materials used in catalysis (Kacimi et al., 2005).

Accordingly, the synthesis and structural characterization of new alluaudite-type phosphates and vanadates within pseudoternary $A_{2} \mathrm{O} / M \mathrm{O} / \mathrm{P}_{2} \mathrm{O}_{5}$ or pseudo-quaternary $A_{2} \mathrm{O} / M \mathrm{O} / \mathrm{Fe}_{2} \mathrm{O}_{3} /$ $\mathrm{P}_{2} \mathrm{O}_{5}$ systems using hydrothermal or solid-state reactions was the focus of our current research. Obtained phases are, for example, $\mathrm{NaMg}_{3}\left(\mathrm{HPO}_{4}\right)_{2}\left(\mathrm{PO}_{4}\right)$ (Ould Saleck et al., 2015), $\mathrm{Na}_{2} \mathrm{Co}_{2} \mathrm{Fe}\left(\mathrm{PO}_{4}\right)_{3}$ (Bouraima et al., 2015) or $\mathrm{Na}_{1.67} \mathrm{Zn}_{1.67-}$ $\mathrm{Fe}_{1.33}\left(\mathrm{PO}_{4}\right)_{3}(\mathrm{Khmiyas}$ et al., 2015). We have also succeeded in preparing the first vanadate-based alluaudite-type phase $\left(\mathrm{Na}_{0.70}\right)\left(\mathrm{Na}_{0.70}, \mathrm{Mn}_{0.30}\right)\left(\mathrm{Fe}^{\mathrm{III}}, \mathrm{Fe}^{\mathrm{II}}\right)_{2} \mathrm{Fe}^{\mathrm{II}}\left(\mathrm{VO}_{4}\right)_{3}$ (Benhsina et al., 


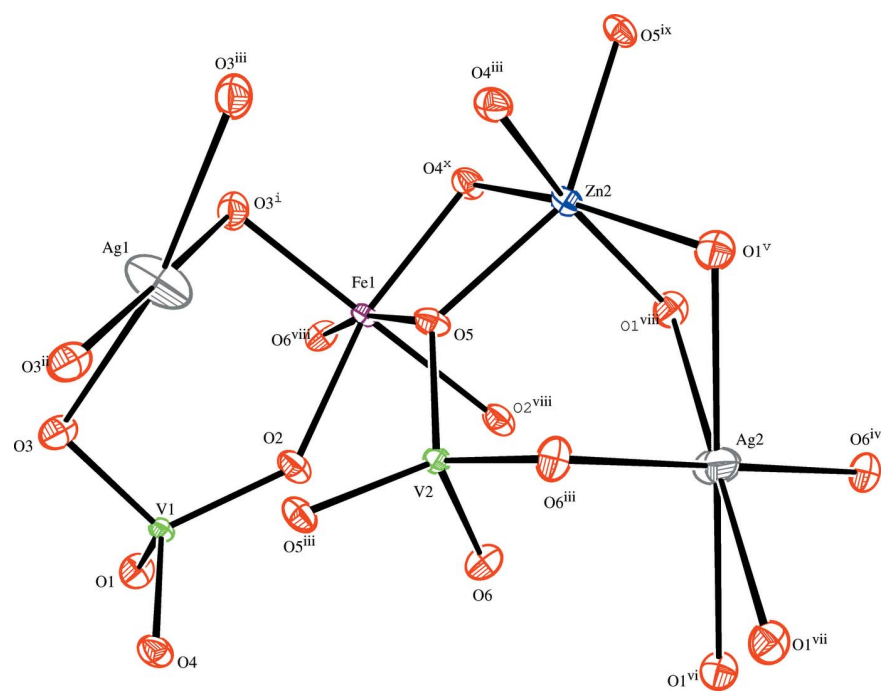

Figure 1

The principal building units in the structure of the title compound. Displacement ellipsoids are drawn at the $50 \%$ probability level. [Symmetry codes: (i) $x,-y+1, z-\frac{1}{2}$; (ii) $-x+1,-y+1,-z+2$; (iii) $-x+1, y,-z+\frac{3}{2}$; (iv) $x,-y, z-\frac{1}{2}$; (v) $x+\frac{1}{2},-y+\frac{1}{2}, z-\frac{1}{2}$; (vi) $-x+\frac{1}{2}, y-\frac{1}{2}$, $-z+\frac{3}{2}$; (vii) $x+\frac{1}{2}, y-\frac{1}{2}, z ;$ (viii) $-x+\frac{1}{2},-y+\frac{1}{2},-z+1$; (ix) $-x+1, y,-z+\frac{1}{2}$; (x) $x, y, z-1$.

2016). A second alluaudite-type vanadate with composition $\mathrm{Na}_{2}\left(\mathrm{Fe}^{\mathrm{III}} / \mathrm{Co}^{\mathrm{II}}\right)_{2} \mathrm{Co}^{\mathrm{II}}\left(\mathrm{VO}_{4}\right)_{3}$ was prepared by Hadouchi et al. (2016) shortly afterwards.

In this context, the current exploration of $A_{2} \mathrm{O} / M \mathrm{O} / \mathrm{Fe}_{2} \mathrm{O}_{3} /$ $\mathrm{V}_{2} \mathrm{O}_{5}$ systems, where $A$ is a monovalent cation and $M$ a divalent cation, led to another vandanate with alluaudite-type structure, namely $\mathrm{Ag}_{2} \mathrm{Zn}_{2} \mathrm{Fe}\left(\mathrm{VO}_{4}\right)_{3}$. Its synthesis and crystal structure are reported in this article.

\section{Structural commentary}

The principal building units of the crystal structure of the new member of the alluaudite-type family are represented in Fig. 1. All atoms are in general positions except for four atoms that are located on special positions. Ag1 is located on an inversion centre (Wyckoff position 4b), and Ag2 as well as Zn2 and V2 are located on twofold rotation axes (4e) of space group $C 2 / c$. The $M 2$ site is in a general position (8f) and statistically occupied by $\mathrm{Fe} 1$ and $\mathrm{Zn} 1$ atoms that are octahedrally surrounded by $\mathrm{O}$ atoms. Such a partial cationic disorder was

a

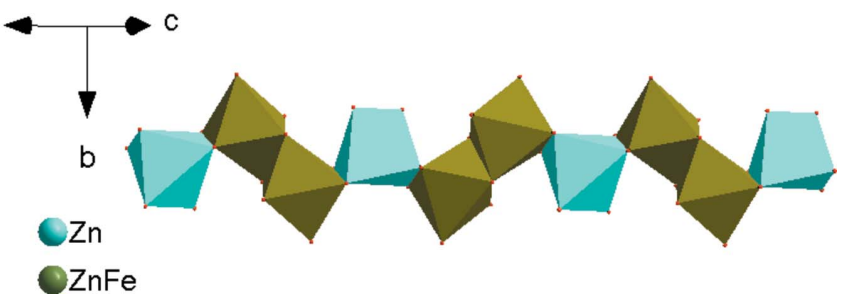

Figure 2

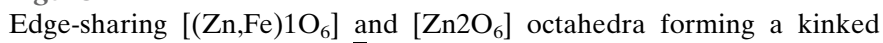

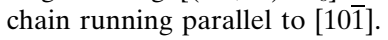

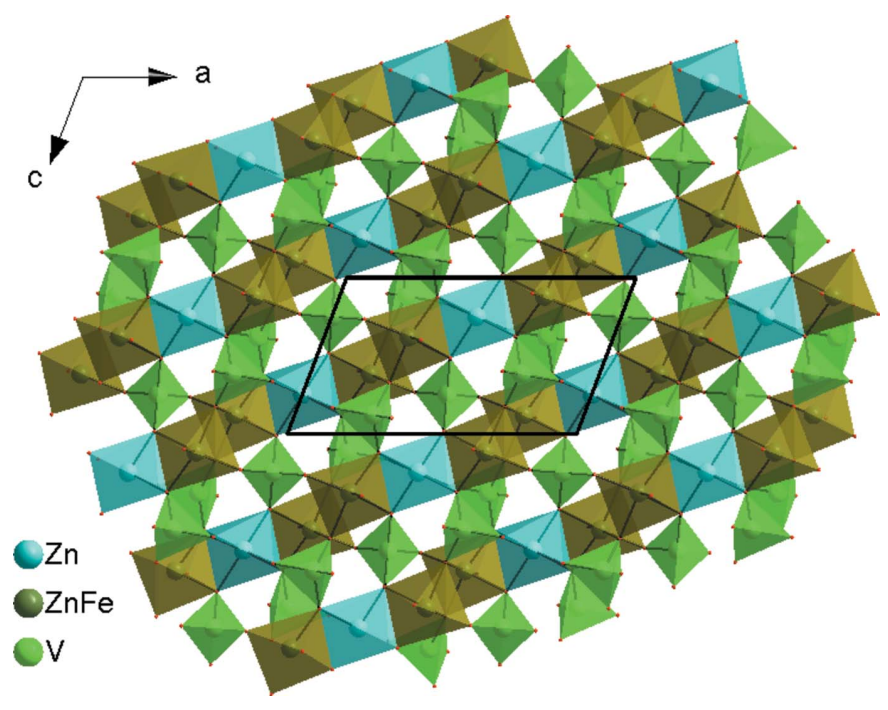

Figure 3

A layer perpendicular to (010), resulting from the connection of chains via the vertices of $\mathrm{VO}_{4}$ tetrahedra and $\left[\mathrm{ZnO}_{6}\right]$ octahedra.

also reported for the cobalt homologue $\mathrm{Na}_{2}\left(\mathrm{Fe}^{\mathrm{III}} / \mathrm{Co}^{\mathrm{II}}\right)_{2^{-}}$ $\mathrm{Co}^{\mathrm{II}}\left(\mathrm{VO}_{4}\right)_{3}$ (Hadouchi et al., 2016).

The crystal structure of $\mathrm{Ag}_{2} \mathrm{Zn}_{2} \mathrm{Fe}\left(\mathrm{VO}_{4}\right)_{3}$ is made up from $\left[(\mathrm{Zn}, \mathrm{Fe}) 1_{2} \mathrm{O}_{10}\right]$ dimers, resulting from edge-sharing $\left[(\mathrm{Zn}, \mathrm{Fe}) 1 \mathrm{O}_{6}\right]$ octahedra, that are connected by a common

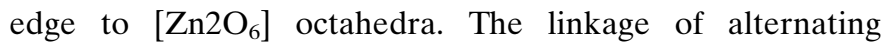
$\left[(\mathrm{Zn}, \mathrm{Fe}) 1_{2} \mathrm{O}_{10}\right]$ and $\left[\mathrm{Zn} 2 \mathrm{O}_{6}\right]$ units leads to infinite zigzag

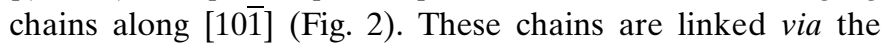
vertices of $\mathrm{VO}_{4}$ tetrahedra into layers parallel to (010), as

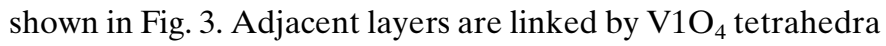
into a three-dimensional framework structure that delimits two types of channels in which the $\mathrm{Ag}^{\mathrm{I}}$ cations reside (Fig. 4). The Ag1 site is located in one channel and is surrounded by

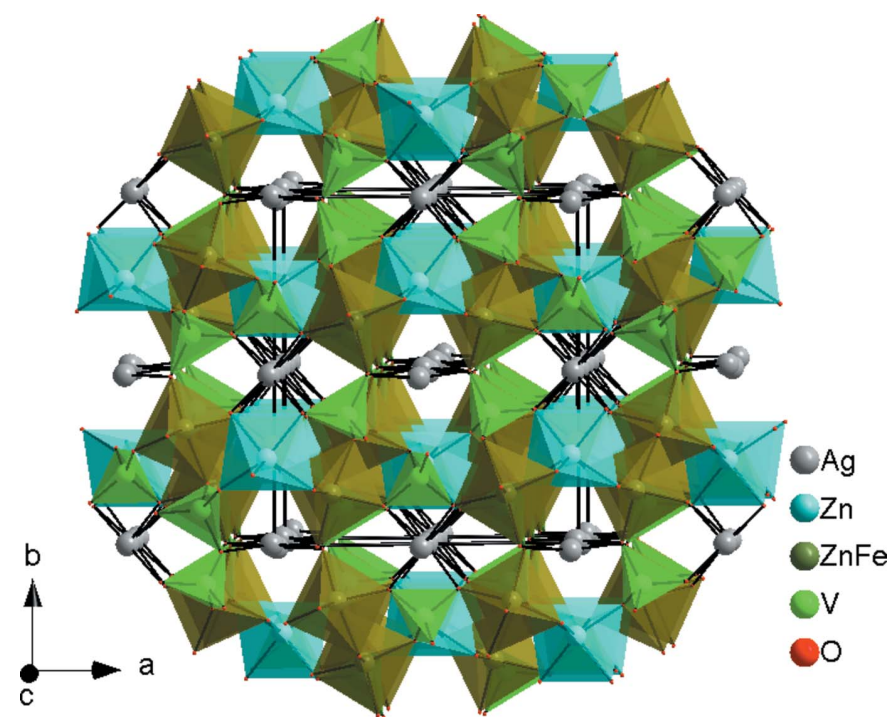

Figure 4

Polyhedral representation of $\mathrm{Ag}_{2} \mathrm{Zn}_{2} \mathrm{Fe}\left(\mathrm{VO}_{4}\right)_{3}$ showing the channels running parallel to the [001] direction. 
Table 1

Experimental details.

\begin{tabular}{|c|c|}
\hline \multicolumn{2}{|l|}{ Crystal data } \\
\hline Chemical formula & $\mathrm{Ag}_{2} \mathrm{Zn}_{2} \mathrm{Fe}\left(\mathrm{VO}_{4}\right)_{3}$ \\
\hline$M_{\mathrm{r}}$ & 747.15 \\
\hline Crystal system, space group & Monoclinic, $C 2 / c$ \\
\hline Temperature (K) & 296 \\
\hline$a, b, c(\AA)$ & 11.8025 (2), 12.9133 (2), 6.8000 (1) \\
\hline$\beta\left(^{\circ}\right)$ & $110.759(1)$ \\
\hline$V\left(\AA^{3}\right)$ & $969.10(3)$ \\
\hline$Z$ & 4 \\
\hline Radiation type & Мо $K \alpha$ \\
\hline$\mu\left(\mathrm{mm}^{-1}\right)$ & 13.09 \\
\hline Crystal size $(\mathrm{mm})$ & $0.31 \times 0.26 \times 0.20$ \\
\hline \multicolumn{2}{|l|}{ Data collection } \\
\hline Diffractometer & Bruker X8 APEX \\
\hline Absorption correction & $\begin{array}{l}\text { Multi-scan (SADABS; Krause et } \\
\quad \text { al., 2015) }\end{array}$ \\
\hline$T_{\min }, T_{\max }$ & $0.596,0.748$ \\
\hline $\begin{array}{l}\text { No. of measured, independent and } \\
\text { observed }[I>2 \sigma(I)] \text { reflections }\end{array}$ & $30791,2662,2437$ \\
\hline$R_{\text {int }}$ & 0.042 \\
\hline$(\sin \theta / \lambda)_{\max }\left(\AA^{-1}\right)$ & 0.869 \\
\hline \multicolumn{2}{|l|}{ Refinement } \\
\hline$R\left[F^{2}>2 \sigma\left(F^{2}\right)\right], w R\left(F^{2}\right), S$ & $0.021,0.048,1.13$ \\
\hline No. of reflections & 2662 \\
\hline No. of parameters & 95 \\
\hline$\Delta \rho_{\max }, \Delta \rho_{\min }\left(\mathrm{e} \AA^{-3}\right)$ & $1.36,-2.41$ \\
\hline
\end{tabular}

Computer programs: APEX2 and SAINT (Bruker, 2014), SHELXT2014 (Sheldrick, 2015a), SHELXL2014 (Sheldrick, 2015b), ORTEP-3 for Windows (Farrugia, 2012), DIAMOND (Brandenburg, 2006) and publCIF (Westrip, 2010).

four oxygen atoms, whereas the $\mathrm{Ag} 2$ site in the second channel is surrounded by six oxygen atoms.

The calculated bond-valences sums (Brown \& Altermatt, 1985) of the atoms in the structure are in the expected ranges for $\mathrm{Ag}^{\mathrm{I}}, \mathrm{Zn}^{\mathrm{II}}, \mathrm{Fe}^{\mathrm{III}}$ and $\mathrm{V}^{\mathrm{V}}$ and are as follows (values in valence units): Ag1 (0.83), Ag2 (1.11), Zn1 (1.95), Zn2 (2.20), Fe1 (2.67), V1 (4.98) and V2 (4.93); values of oxygen atoms range between 1.90 and 2.01 valence units.

\section{Database Survey}

Over the last twenty years, many synthetic alluaudite-type phosphates, arsenates, sulfates and molybdates have been reported, such as $\mathrm{NaMnFe}_{2}\left(\mathrm{PO}_{4}\right)_{3}$ used as the positive electrode in sodium and lithium batteries (Trad et al., 2010; Kim et al., 2014; Huang et al., 2015), $\mathrm{Na}_{2.44} \mathrm{Mn}_{1.79}\left(\mathrm{SO}_{4}\right)_{3}$ used as a potential high-voltage cathode material ( $c a 4.4 \mathrm{~V}$ ) for sodium batteries (Dwibedi et al., 2015), $\mathrm{K}_{0.13} \mathrm{Na}_{3.87} \mathrm{Mg}\left(\mathrm{MoO}_{4}\right)_{3}$ as a promising compound for developing new materials with high ionic conductivity (Ennajeh et al., 2015), or $\mathrm{NaZn}_{3}\left(\mathrm{AsO}_{4}\right)$ $\left(\mathrm{AsO}_{3} \mathrm{OH}\right)_{2}$ (Đorđević et al., 2015).

\section{Synthesis and crystallization}

$\mathrm{Ag}_{2} \mathrm{Zn}_{2} \mathrm{Fe}\left(\mathrm{VO}_{4}\right)_{3}$ was prepared by a solid-state reaction. A stoichiometric amount of silver nitrate $\left(\mathrm{AgNO}_{3}\right)$, zinc acetate $\left(\mathrm{Zn}\left(\mathrm{CH}_{3} \mathrm{COO}\right)_{2} \cdot 2 \mathrm{H}_{2} \mathrm{O}\right)$, iron nitrate $\left.\left(\mathrm{Fe}\left(\mathrm{NO}_{3}\right)_{3}\right) \cdot 9 \mathrm{H}_{2} \mathrm{O}\right)$ and vanadium oxide $\left(\mathrm{V}_{2} \mathrm{O}_{5}\right)$ was employed in the molar ratio $\mathrm{Ag}$ :
$\mathrm{Zn}: \mathrm{Fe}: \mathrm{V}=2: 2: 1: 3$ and put into a platinum cruicible. After different heat treatments at lower temperatures to remove water and other voliatile gaseous products, the reaction mixture was melted at $1033 \mathrm{~K}$ for 30 minutes, followed by slow cooling with a $5 \mathrm{~K} \mathrm{~h}^{-1}$ rate to room temperature. The resulting product contained parallelepipedic orange crystals corresponding to the studied title vanadate. In addition, small blocklike crystals with poor quality and unidentified by X-ray powder diffraction were present.

\section{Refinement}

Crystal data, data collection and structure refinement details are summarized in Table 1 . The remaining maximum and minimum electron density peaks in the final Fourier map are $0.40 \AA$ away from Fe1 and $0.62 \AA$ from Ag1, respectively. Due to charge neutrality, sites $\mathrm{Zn} 1$ and Fe2 were modelled as statistically occupied, assuming a trivalent oxidation state for the iron site.

\section{Acknowledgements}

The authors thank the Unit of Support for Technical and Scientific Research (UATRS, CNRST) for the X-ray measurements.

\section{Funding information}

The authors thank Mohammed V University, Rabat, Morocco, for financial support.

\section{References}

Benhsina, E., Assani, A., Saadi, M. \& El Ammari, L. (2016). Acta Cryst. E72, 220-222.

Bouraima, A., Assani, A., Saadi, M., Makani, T. \& El Ammari, L. (2015). Acta Cryst. E71, 558-560.

Brandenburg, K. (2006). DIAMOND. Crystal Impact GbR, Bonn, Germany.

Brown, I. D. \& Altermatt, D. (1985). Acta Cryst. B41, 244-247.

Bruker (2014). APEX2 and SAINT. Bruker AXS Inc., Madison, Wisconsin, USA.

Đorđević, T., Wittwer, A. \& Krivovichev, S. V. (2015). Eur. J. Mineral. 27, 559-573.

Dwibedi, D., Araujo, R. B., Chakraborty, S., Shanbogh, P. P., Sundaram, N. G., Ahuja, R. \& Barpanda, P. (2015). J. Mater. Chem. A, 3, 18564-18571.

Ennajeh, I., Georges, S., Smida, Y. B., Guesmi, A., Zid, M. F. \& Boughazala, H. (2015). RSC Adv. 5, 38918-38925.

Farrugia, L. J. (2012). J. Appl. Cryst. 45, 849-854.

Hadouchi, M., Assani, A., Saadi, M. \& El Ammari, L. (2016). Acta Cryst. E72, 1017-1020.

Hatert, F. (2008). J. Solid State Chem. 181, 1258-1272.

Huang, W., Li, B., Saleem, M. F., Wu, X., Li, J., Lin, J., Xia, D., Chu, W. \& Wu, Z. (2015). Chem. Eur. J. 21, 851-860.

Kacimi, M., Ziyad, M. \& Hatert, F. (2005). Mater. Res. Bull. 40, 682693.

Khmiyas, J., Assani, A., Saadi, M. \& El Ammari, L. (2015). Acta Cryst. E71, 690-692.

Kim, J., Kim, H., Park, K.-Y., Park, Y.-U., Lee, S., Kwon, H.-S., Yoo, H.-I. \& Kang, K. (2014). J. Mater. Chem. A, 2, 8632-8636.

Korzenski, M. B., Schimek, G. L., Kolis, J. W. \& Long, G. J. (1998). J. Solid State Chem. 139, 152-160. 
Krause, L., Herbst-Irmer, R., Sheldrick, G. M. \& Stalke, D. (2015). J. Appl. Cryst. 48, 3-10.

Moore, P. B. (1971). Am. Mineral. 56, 1955-1975.

Ould Saleck, A., Assani, A., Saadi, M., Mercier, C., Follet, C. \& El Ammari, L. (2015). Acta Cryst. E71, 813-815.
Sheldrick, G. M. (2015a). Acta Cryst. A71, 3-8.

Sheldrick, G. M. (2015b). Acta Cryst. C71, 3-8.

Trad, K., Carlier, D., Croguennec, L., Wattiaux, A., Ben Amara, M. \& Delmas, C. (2010). Chem. Mater. 22, 5554-5562.

Westrip, S. P. (2010). J. Appl. Cryst. 43, 920-925. 


\section{supporting information}

Acta Cryst. (2018). E74, 1155-1158 [https://doi.org/10.1107/S205698901801071X]

Crystal structure of disilver(I) dizinc(II) iron(III) tris(orthovanadate) with an alluaudite-type structure

Nour El Houda Lamsakhar, Mohammed Zriouil, Abderrazzak Assani, Mohamed Saadi and Lahcen El Ammari

Computing details

Data collection: APEX2 (Bruker, 2014); cell refinement: SAINT (Bruker, 2014); data reduction: SAINT (Bruker, 2014); program(s) used to solve structure: SHELXT2014 (Sheldrick, 2015a); program(s) used to refine structure: SHELXL2014 (Sheldrick, 2015b); molecular graphics: ORTEP-3 for Windows (Farrugia, 2012) and DIAMOND (Brandenburg, 2006); software used to prepare material for publication: publCIF (Westrip, 2010).

Disilver(I) dizinc(II) iron(III) tris(orthovanadate)

Crystal data

$\mathrm{Ag}_{2} \mathrm{Zn}_{2} \mathrm{Fe}\left(\mathrm{VO}_{4}\right)_{3}$

$M_{r}=747.15$

Monoclinic, $C 2 / c$

$a=11.8025(2) \AA$

$b=12.9133(2) \AA$

$c=6.8000(1) \AA$

$\beta=110.759(1)^{\circ}$

$V=969.10(3) \AA^{3}$

$Z=4$

\section{Data collection}

Bruker X8 APEX

diffractometer

Radiation source: fine-focus sealed tube

Graphite monochromator

$\varphi$ and $\omega$ scans

Absorption correction: multi-scan

(SADABS; Krause et al., 2015)

$T_{\min }=0.596, T_{\max }=0.748$

Refinement

Refinement on $F^{2}$

Least-squares matrix: full

$R\left[F^{2}>2 \sigma\left(F^{2}\right)\right]=0.021$

$w R\left(F^{2}\right)=0.048$

$S=1.13$

2662 reflections

95 parameters
$F(000)=1380$

$D_{\mathrm{x}}=5.121 \mathrm{Mg} \mathrm{m}^{-3}$

Mo $K \alpha$ radiation, $\lambda=0.71073 \AA$

Cell parameters from 2662 reflections

$\theta=2.4-38.1^{\circ}$

$\mu=13.09 \mathrm{~mm}^{-1}$

$T=296 \mathrm{~K}$

Parallelepiped, orange

$0.31 \times 0.26 \times 0.20 \mathrm{~mm}$

30791 measured reflections

2662 independent reflections

2437 reflections with $I>2 \sigma(I)$

$R_{\text {int }}=0.042$

$\theta_{\text {max }}=38.1^{\circ}, \theta_{\min }=2.4^{\circ}$

$h=-18 \rightarrow 20$

$k=-22 \rightarrow 22$

$l=-11 \rightarrow 9$

0 restraints

$w=1 /\left[\sigma^{2}\left(F_{\mathrm{o}}^{2}\right)+(0.0126 P)^{2}+4.2342 P\right]$

where $P=\left(F_{\mathrm{o}}{ }^{2}+2 F_{\mathrm{c}}{ }^{2}\right) / 3$

$(\Delta / \sigma)_{\max }=0.001$

$\Delta \rho_{\max }=1.36 \mathrm{e} \AA^{-3}$

$\Delta \rho_{\min }=-2.41$ e $\AA^{-3}$ 
Extinction correction: SHELXL2016

(Sheldrick, 2015b),

$\mathrm{Fc}^{*}=\mathrm{kFc}\left[1+0.001 \mathrm{xFc}^{2} \lambda^{3} / \sin (2 \theta)\right]^{-1 / 4}$

Extinction coefficient: 0.00163 (6)

\section{Special details}

Geometry. All esds (except the esd in the dihedral angle between two 1.s. planes) are estimated using the full covariance matrix. The cell esds are taken into account individually in the estimation of esds in distances, angles and torsion angles; correlations between esds in cell parameters are only used when they are defined by crystal symmetry. An approximate (isotropic) treatment of cell esds is used for estimating esds involving l.s. planes.

Fractional atomic coordinates and isotropic or equivalent isotropic displacement parameters $\left(\AA^{2}\right)$

\begin{tabular}{llllll}
\hline & $x$ & $y$ & $z$ & $U_{\text {iso }} * / U_{\text {eq }}$ & Occ. $(<1)$ \\
\hline Ag1 & 0.500000 & $0.49090(3)$ & 0.750000 & $0.02736(7)$ & \\
Ag2 & 0.500000 & 0.000000 & 0.500000 & $0.02115(6)$ & \\
Zn2 & 0.500000 & $0.23529(2)$ & 0.250000 & $0.00945(6)$ & \\
Zn1 & $0.29222(2)$ & $0.34062(2)$ & $0.38041(3)$ & $0.00652(5)$ & 0.5 \\
Fe1 & $0.29222(2)$ & $0.34062(2)$ & $0.38041(3)$ & $0.00652(5)$ & 0.5 \\
V1 & $0.27045(3)$ & $0.38683(2)$ & $0.88206(4)$ & $0.00612(5)$ & \\
V2 & 0.500000 & $0.20643(3)$ & 0.750000 & $0.00602(6)$ & \\
O1 & $0.12116(12)$ & $0.39616(11)$ & $0.8338(2)$ & $0.0128(2)$ & \\
O2 & $0.28524(13)$ & $0.31700(11)$ & $0.6746(2)$ & $0.0124(2)$ & \\
O3 & $0.33803(14)$ & $0.50767(11)$ & $0.8997(2)$ & $0.0139(2)$ & \\
O4 & $0.33926(12)$ & $0.32576(11)$ & $1.1233(2)$ & $0.0112(2)$ & \\
O5 & $0.46319(12)$ & $0.27705(11)$ & $0.5152(2)$ & $0.0099(2)$ & \\
O6 & $0.38484(12)$ & $0.12416(10)$ & $0.7343(2)$ & $0.0115(2)$ &
\end{tabular}

Atomic displacement parameters $\left(\AA^{2}\right)$

\begin{tabular}{lllllll}
\hline & $U^{11}$ & $U^{22}$ & $U^{33}$ & $U^{12}$ & $U^{13}$ & $U^{23}$ \\
\hline Ag1 & $0.01209(9)$ & $0.05204(18)$ & $0.01674(11)$ & 0.000 & $0.00362(8)$ & 0.000 \\
Ag2 & $0.03519(13)$ & $0.01557(9)$ & $0.01224(9)$ & $-0.01110(8)$ & $0.00786(9)$ & $-0.00296(7)$ \\
Zn2 & $0.00905(11)$ & $0.01104(12)$ & $0.00942(12)$ & 0.000 & $0.00472(9)$ & 0.000 \\
Zn1 & $0.00607(8)$ & $0.00863(9)$ & $0.00535(9)$ & $0.00074(6)$ & $0.00264(6)$ & $0.00062(6)$ \\
Fe1 & $0.00607(8)$ & $0.00863(9)$ & $0.00535(9)$ & $0.00074(6)$ & $0.00264(6)$ & $0.00062(6)$ \\
V1 & $0.00655(10)$ & $0.00709(10)$ & $0.00474(10)$ & $0.00040(8)$ & $0.00202(8)$ & $0.00021(8)$ \\
V2 & $0.00649(14)$ & $0.00644(14)$ & $0.00448(14)$ & 0.000 & $0.00112(11)$ & 0.000 \\
O1 & $0.0095(5)$ & $0.0130(5)$ & $0.0160(6)$ & $0.0017(4)$ & $0.0045(5)$ & $0.0008(5)$ \\
O2 & $0.0140(6)$ & $0.0155(6)$ & $0.0082(5)$ & $0.0017(5)$ & $0.0046(4)$ & $-0.0004(4)$ \\
O3 & $0.0149(6)$ & $0.0116(5)$ & $0.0158(6)$ & $-0.0010(4)$ & $0.0061(5)$ & $0.0017(5)$ \\
O4 & $0.0123(5)$ & $0.0133(5)$ & $0.0078(5)$ & $0.0037(4)$ & $0.0034(4)$ & $0.0019(4)$ \\
O5 & $0.0090(5)$ & $0.0135(5)$ & $0.0075(5)$ & $0.0019(4)$ & $0.0035(4)$ & $0.0027(4)$ \\
O6 & $0.0087(5)$ & $0.0103(5)$ & $0.0136(6)$ & $-0.0007(4)$ & $0.0019(4)$ & $0.0016(4)$ \\
& & & & & & \\
\hline
\end{tabular}

Geometric parameters $\left(\AA,{ }^{\circ}\right)$

\begin{tabular}{llll}
\hline $\mathrm{Ag} 1-\mathrm{O} 3^{\mathrm{i}}$ & $2.4699(15)$ & $\mathrm{Zn} 2-\mathrm{O}^{\mathrm{v}}$ & $2.1619(15)$ \\
$\mathrm{Ag} 1-\mathrm{O}^{\mathrm{ii}}$ & $2.4699(16)$ & $\mathrm{Zn} 1-\mathrm{O}^{\text {viii }}$ & $2.0068(14)$
\end{tabular}




$$
\begin{aligned}
& \text { Ag1-O3 }{ }^{\text {iii }} \\
& \mathrm{Ag} 1-\mathrm{O} 3 \\
& \mathrm{Ag} 2-\mathrm{O}^{\text {iv }} \\
& \text { Ag2-O6 }{ }^{\text {iii }} \\
& \mathrm{Ag} 2-\mathrm{O} 1^{\mathrm{v}} \\
& \mathrm{Ag} 2-\mathrm{O}^{\text {vi }} \\
& \text { Ag2-O1 }{ }^{\text {vii }} \\
& \mathrm{Ag} 2-\mathrm{O}^{\text {viii }} \\
& \mathrm{Zn} 2-\mathrm{O} 5^{\mathrm{ix}} \\
& \mathrm{Zn} 2-\mathrm{O} 5 \\
& \mathrm{Zn} 2-\mathrm{O} 4^{\mathrm{iii}} \\
& \mathrm{Zn} 2-\mathrm{O} 4^{\mathrm{x}} \\
& \mathrm{Zn} 2-\mathrm{O} 1^{\text {viii }} \\
& \mathrm{O}^{\mathrm{i}}-\mathrm{Ag} 1-\mathrm{O}^{\mathrm{ii}} \\
& \mathrm{O} 3^{\mathrm{i}}-\mathrm{Ag} 1-\mathrm{O}^{\mathrm{iii}} \\
& \mathrm{O} 3^{\mathrm{ii}}-\mathrm{Ag} 1-\mathrm{O} 3^{\mathrm{iii}} \\
& \mathrm{O} 3-\mathrm{Ag} 1-\mathrm{O} 3 \\
& \mathrm{O} 3{ }^{\mathrm{ii}}-\mathrm{Ag} 1-\mathrm{O} 3 \\
& \mathrm{O} 3{ }^{\mathrm{iii}-\mathrm{Ag} 1-\mathrm{O} 3} \\
& \text { O6 }{ }^{\text {iv }}-\mathrm{Ag} 2-\mathrm{O}^{\text {iii }} \\
& \mathrm{O}^{\mathrm{iv}}-\mathrm{Ag} 2-\mathrm{O}^{\mathrm{v}}
\end{aligned}
$$

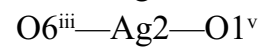

$$
\begin{aligned}
& \mathrm{O}^{\mathrm{iv}}-\mathrm{Ag} 2-\mathrm{O}^{\mathrm{vi}} \\
& \mathrm{O} 6^{\mathrm{iii}}-\mathrm{Ag} 2-\mathrm{O}^{\mathrm{vi}} \\
& \mathrm{O} 1^{\mathrm{v}}-\mathrm{Ag} 2-\mathrm{O} 1^{\mathrm{vi}} \\
& \mathrm{O} 6^{\mathrm{iv}}-\mathrm{Ag} 2-\mathrm{O} 1^{\text {vii }} \\
& \mathrm{O}^{\mathrm{iii}}-\mathrm{Ag} 2-\mathrm{O} 1^{\mathrm{vii}} \\
& \mathrm{O} 1^{\mathrm{v}}-\mathrm{Ag} 2-\mathrm{O} 1^{\mathrm{vii}} \\
& \mathrm{O} 1^{\mathrm{vi}}-\mathrm{Ag} 2-\mathrm{O} 1^{\mathrm{vii}} \\
& \mathrm{O}^{\mathrm{iv}}-\mathrm{Ag} 2-\mathrm{O}^{\text {viii }} \\
& \mathrm{O}^{\mathrm{iii}}-\mathrm{Ag} 2-\mathrm{O}^{\text {viii }} \\
& \mathrm{O} 1^{\mathrm{v}}-\mathrm{Ag} 2-\mathrm{O} 1^{\text {viii }} \\
& \mathrm{O} 1^{\mathrm{vi}}-\mathrm{Ag} 2-\mathrm{O} 1^{\text {viii }}
\end{aligned}
$$

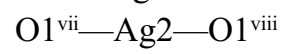

$$
\begin{aligned}
& \mathrm{O} 5{ }^{\mathrm{ix}}-\mathrm{Zn} 2-\mathrm{O} 5 \\
& \mathrm{O} 5^{\mathrm{ix}}-\mathrm{Zn} 2-\mathrm{O} 4^{\mathrm{iii}} \\
& \mathrm{O} 5-\mathrm{Zn} 2-\mathrm{O} 4^{\mathrm{iii}} \\
& \mathrm{O} 5^{\mathrm{ix}}-\mathrm{Zn} 2-\mathrm{O} 4^{\mathrm{x}} \\
& \mathrm{O} 5-\mathrm{Zn} 2-\mathrm{O} 4^{\mathrm{x}} \\
& \mathrm{O} 4^{\mathrm{iii}}-\mathrm{Zn} 2-\mathrm{O}^{\mathrm{x}} \\
& \mathrm{O}^{\text {ix }}-\mathrm{Zn} 2-\mathrm{O} 1^{\text {viii }} \\
& \mathrm{O} 5-\mathrm{Zn} 2-\mathrm{O} 1^{\text {viii }}
\end{aligned}
$$

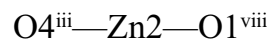

$2.4734(16)$

$2.4734(16)$

$2.4374(14)$

2.4374 (14)

$2.5032(15)$

$2.5032(15)$

$2.5873(14)$

$2.5873(14)$

2.0704 (14)

2.0705 (14)

$2.1325(13)$

$2.1325(13)$

$2.1619(15)$

$179.15(7)$

$92.83(5)$

$87.10(5)$

$87.10(5)$

$92.83(5)$

$169.95(7)$

$180.00(6)$

$105.99(5)$

$74.01(5)$

$74.01(5)$

$105.99(5)$

$180.00(6)$

$107.39(5)$

$72.61(5)$

$116.56(6)$

$63.44(6)$

$72.61(5)$

$107.39(5)$

$63.44(6)$

$116.56(6)$

180.0

$149.81(8)$

$77.17(5)$

$86.37(5)$

$86.37(5)$

$77.17(5)$

$113.56(8)$

$107.36(5)$

96.35 (6)

$161.31(5)$

$$
\begin{aligned}
& \mathrm{Zn} 1-\mathrm{O}^{\mathrm{x}} \\
& \mathrm{Zn} 1-3^{\mathrm{i}} \\
& \mathrm{Zn} 1-\mathrm{O} 2 \\
& \mathrm{Zn} 1-\mathrm{O} 5 \\
& \mathrm{Zn} 1-\mathrm{O} 2^{\mathrm{viii}} \\
& \mathrm{V} 1-\mathrm{O} 1 \\
& \mathrm{~V} 1-\mathrm{O} 2 \\
& \mathrm{~V} 1-\mathrm{O} 3 \\
& \mathrm{~V} 1-\mathrm{O} 4 \\
& \mathrm{~V} 2-\mathrm{O} 6 \\
& \mathrm{~V} 2-\mathrm{O} 6^{\mathrm{iii}} \\
& \mathrm{V} 2-\mathrm{O} 5^{\mathrm{iii}} \\
& \mathrm{V} 2-\mathrm{O} 5
\end{aligned}
$$

2.0222 (14)

2.0241 (15)

2.0540 (14)

2.0675 (13)

2.2082 (15)

1.6784 (14)

1.7343 (14)

$1.7372(15)$

1.7402 (13)

1.6984 (14)

1.6984 (14)

1.7544 (13)

1.7544 (13)

$107.36(5)$

85.01 (5)

$161.31(5)$

76.52 (7)

104.63 (6)

91.33 (6)

89.94 (6)

$90.95(6)$

161.09 (6)

$100.52(6)$

$168.70(6)$

$79.73(5)$

$99.16(6)$

83.05 (5)

80.30 (5)

89.43 (5)

171.16 (6)

82.59 (6)

89.40 (5)

$106.24(7)$

111.93 (7)

$110.32(7)$

108.88 (7)

112.54 (7)

107.01 (7)

$102.56(10)$

$108.46(7)$

$109.48(6)$

109.48 (6)

108.47 (7) 
supporting information

$\begin{array}{llll}\mathrm{O}^{\mathrm{x}}-\mathrm{Zn} 2-\mathrm{O}^{\mathrm{viii}} & 85.01(5) & \mathrm{O} 5^{\mathrm{iii}}-\mathrm{V} 2-\mathrm{O} 5 & 117.36(9) \\ \mathrm{O}^{5}{ }^{\mathrm{ix}}-\mathrm{Zn} 2-\mathrm{O}^{\mathrm{v}} & 96.35(6) & & \end{array}$

Symmetry codes: (i) $x,-y+1, z-1 / 2$; (ii) $-x+1,-y+1,-z+2$; (iii) $-x+1, y,-z+3 / 2$; (iv) $x,-y, z-1 / 2$; (v) $x+1 / 2,-y+1 / 2, z-1 / 2$; (vi) $-x+1 / 2, y-1 / 2,-z+3 / 2$; (vii) $x+1 / 2, y-1 / 2, z$; (viii) $-x+1 / 2,-y+1 / 2,-z+1$; (ix) $-x+1, y,-z+1 / 2$; (x) $x, y, z-1$. 